\title{
Case report: left hand Acheiria in 3 newborns within a period of 3 months during the year 2011 in Fallujah general hospital
}

\author{
Samira Telfah Alaani ${ }^{1}$, Abdulkadir Abdulkareem Miri'e ${ }^{2}$ \\ ${ }^{1}$ Pediatric Department, Fallujah General Hospital, Fallujah, Anbar province, Iraq, 00964 \\ ${ }^{2}$ Clinical geneticist, Fallujah General Hospital, Fallujah, Anbar province, Iraq, 00964
}

\section{Email address:}

samiraalaani@hotmail.com(S. T. Alaani),abd69k@yahoo.com(A. A. Miri'e)

\section{To cite this article:}

Samira Telfah Alaani, Abdulkadir Abdulkareem Miri'e. Case Report: Left Hand Acheiria in 3 Newborns within a Period of 3 Months during the Year 2011 in Fallujah General Hospital. Clinical Medicine Research. Vol. 2, No. 4, 2013, pp. 84-88. doi: 10.11648/j.cmr.20130204.19

\begin{abstract}
We present 3 sporadic cases of congenital left hand Acheiria, a rare congenital defect, In one case (male), left hand Acheiria is associated with left radial hemimelia, in the other 2 cases (both are females), there is only congenital absence of left hand ( Acheiria), with completely normal left forearm \& other 3 limbs. The absence of any contributory family or drug history and the normal chromosomal study in the 3 cases lead us to the belief that their occurrence may be linked to environmental contamination. To the best of our knowledge, there is dearth of information on these anomalies in the literatures, \& their management remains achallenge.
\end{abstract}

Keywords: Acheiria, Hemimelia, Fallujah General Hospital

\section{Introduction}

The congenital malformations of upper limbs are not a very frequent, for they affect less than $0.2 \%$ of born babies. Many of these malformations are not severe, have a minor functional impact and may be surgically treated.

The embryologic development of the upper limb takes place in the early stages, presenting a complete differentiation at week 7 of gestation ${ }^{1}$ Usually, if malformation occurs, it might be present by the time the mother confirms her pregnancy.

There are many classifications to categorize the various forms of congenital amputation.

According to the International Federation of Societies for Surgery of the Hand ${ }^{1}$ there are 7 groups of malformations:

failure of formation of parts,

failure of differentiation (separation) of parts,

duplication,

overgrowth,

undergrowth,

congenital constriction band syndrome,

generalized skeletal abnormalities.

Malformations of the first group result in congenital amputations, being the hand and the forearm the most frequent affected. According to the dominating type of malformation, they are divided into:

*Amelia: total absence of upper limb

*Hemimelia: absence of forearm and hand.

*Acheiria: absence of hand.

*Adactilia: absence of metacarpus and phalanges

*Aphalangia: absence of all phalanges.

A previous communication reported results of an epidemiological study in Fallujah/ Iraq, which revealed very high levels of cancer and infant mortality in the period 2005-2010 together with a statistically significant perturbation of the birth sex-ratio of those born from 2005. The authors concluded that some genotoxic exposure had occurred at the same time as the United States-led attacks on Fallujah in April and November $2004^{2}$.

An attempt to investigate the genetic familial causation of four individual cases of congenital anomaly has subsequently been made ${ }^{3}$ the authors concluded that in these cases there was no familial genetic predisposition and that sporadic untargeted effects were responsible, causes which they described as epigenetic ${ }^{3}$.

It was clearly of interest then to further examine the evidence of the increased infants mortality and congenital malformations in Fallujah and to investigate the environment for possible agents that may explain the 
increased incidence of birth defects and cancer rate increases. The environmental aspect of the problem was studied in 2010 when hair sample analysis of 52 elements was carried out on the parents of children diagnosed with congenital anomalies ${ }^{4}$ In addition soil and water samples were analyzed. Results indicated that man-made slightly enriched uranium was present in soil and in the hair of the parents, with a trend of increasing concentration along the hair strands back to 2005 but that no other genotoxic elements were present which could explain the levels of cancer and congenital disease. The question of the incidence and types of congenital anomalies remains ${ }^{4}$.

Then in another pilot study, the numbers of different types of congenital anomalies diagnosed in one of three clinics at Fallujah General Hospital (FGH) over an eleven month period in 2009-2010 have been reported, It was found to be about $144 / 1000$ live births which is much higher than the levels reported in other areas in Iraq \& surrounding countries 5 .

This report details the findings of 2 rare cases of congenital left hand Acheiria , and radial hemimelia \& left hand acheiria in a 3 rd case.

\section{Case Reports}

\subsection{Case 1}

A female patient, from Fallujah -Ameriya subdistrict, born by spontaneous vaginal delivery on 6-4-2011, to a mother whose pregnancy and delivery were uneventful, was presented to the neonatal care unit (NCU) in our hospital with complete absence of her left hand ( congenitally amputated). She is the 9th child in the family, all of the 8 siblings are normal, the youngest one being 8 years old and the patient is the only child born after the year 2004 , detailed history was taken from the mother about her pregnancy, there was no chronic or febrile illness ,no history of epilepsy, no history of x.ray exposure ,no history of drug use like anticonvulsants or any other teratogenic drug. The anomaly was not diagnosed prenatally, the mother $\&$ father are 39 years old and there is no history of consanguinity between them.

There was no prior history of such or other anomaly in the family.

On examination; the left hand was absent (fig.1), there were only remnants of fingers present on the end of the forearm. The left shoulder, elbow, arm and forearm were normal physically and functionally. The other 3 limbs were also completely normal, examination of head, neck, chest including the heart, abdomen and pelvis also revealed no abnormality.

Laboratory work up, including complete blood count, CRP,RBS were normal.

Radiography showed absence of left carpal bones and hand (fig2) with normal forearm bones..

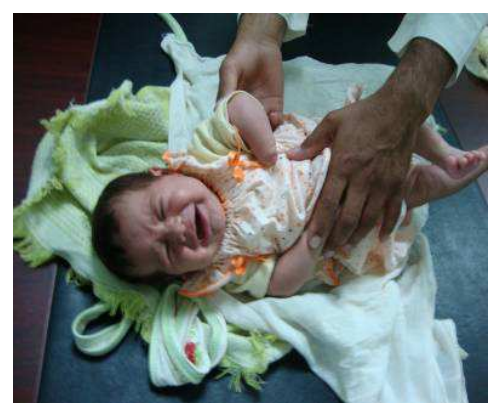

Figure 1. Absent left hand from the level of wrist

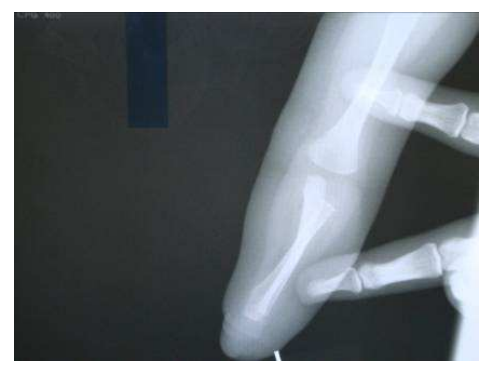

Figure 2. Absent left carpal bones \& hand

\subsection{Case 2}

A female patient delivered by spontaneous vaginal delivery after completing 40 weeks gestation on 26-5-2011, the family live in Fallujah -Nazzal district. She is the 5th child in the family; the other siblings are completely normal and healthy. The mother is 36 years old, she gave a history of regular antenatal care visits, her pregnancy was normal, she gave no history of any chronic illness like hypertension, diabetes or epilepsy, no history of any febrile illness, no history of x.ray exposure or any drug use apart from tonics...she gave history of having 3 miscarriages during the years 2009 and 2010 (before the birth of this child). The anomaly was not diagnosed prenataly. The father's age is 50 years and there is no consanguinity between the two parents.

There is no previous family history of Acheiria. On examination; the left hand was absent from the level of the wrist joint, the left shoulder, elbow, arm \& forearm being completely normal in appearance and function. (fig 3) the contra lateral upper limb and both lower limbs were normal . Head, neck, chest (including heart), abdomen and pelvis were normal. Routine laboratory work up was normal. Radiological examination showed absence of left carpal bone and wrist, the radius and ulna were present and normal.( fig 4). 


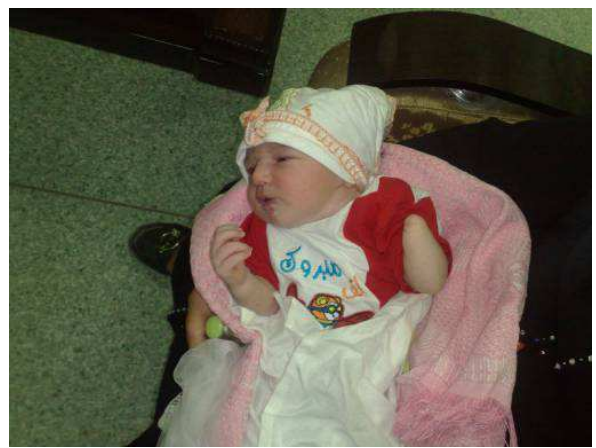

Fig 3. Absent left hand from the level of the wrist joint

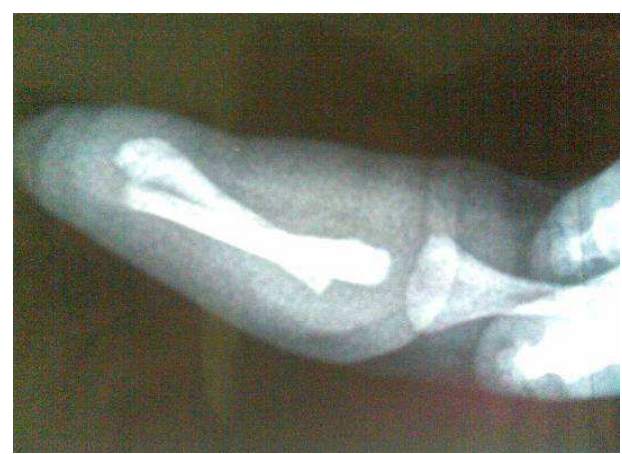

Fig 4. Absent left carpal bone and wrist, the radius and ulna are present and normal

\subsection{Case 3}

A male neonate born by cesarean section after 40 weeks gestation , on 6-7-2011. His family live in Fallujah Saglaweya subdistrict. He is the 5th child in the family, the older 4 siblings are completely normal. The mother is 35 years old \& have gestational hypertension, controlled by diet only, she gave history of hepatitis $\mathrm{B}$ virus infection during pregnancy,there is no history diabetes or epilepsy, no TORCH infection, no history of x.ray exposure or drug use apart from folic acid medication during pregnancy . The anomaly was not detected by prenatal ultrasonographic examination. The father's age is 40 years old and the parents are $3 \mathrm{rd}$ degree relatives. There is no prior family history of Acheiria or any other anomaly.

On examination; the neonate looks completely normal apart from total absence of the left hand from the level of the wrist joint, and there is remnant of fingers at the end of the forearm (fig 5). The contalateral upper limb and both lower limbs were completely normal. Head, neck, chest, heart, abdomen $\&$ pelvis were normal.

Laboratory work up including CBC, CRP, RBS, TSB were normal, HBs Ag was negative.

Radiological examination showed absence of left carpal bones and hand, and also absence of left Radius (left radial hemimelia) (fig 6).

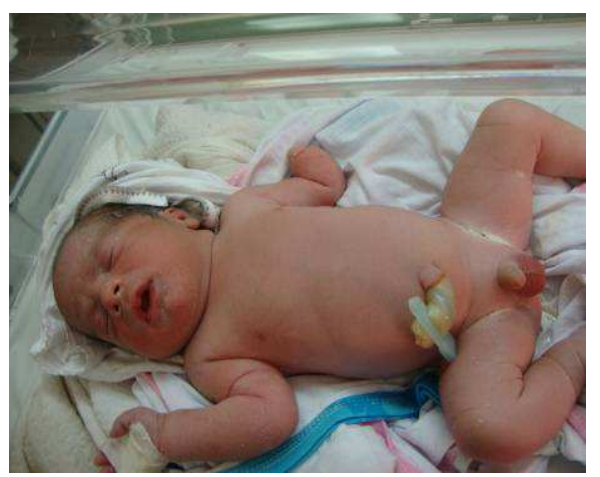

Fig 5. Total absence of the left hand from the level of the wrist joint, and there is remnant of fingers at the end of the forearm

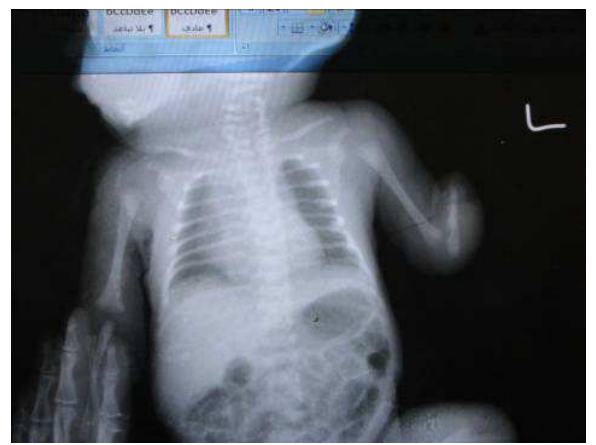

Fig 6. Absence of left carpal bones and hand, and left radial hemimelia

Chromosomal study for the 3 cases was done and revealed no abnormality ( fig 7,8,9) .

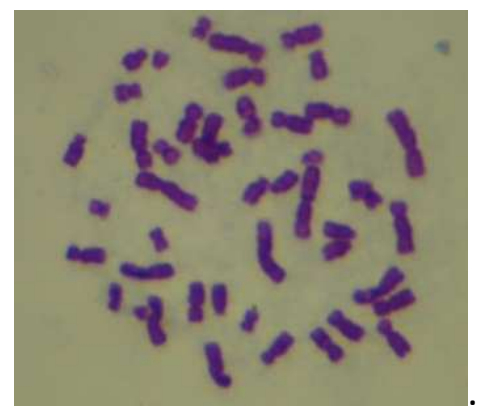

Fig 7. Case 1 chromosomal study

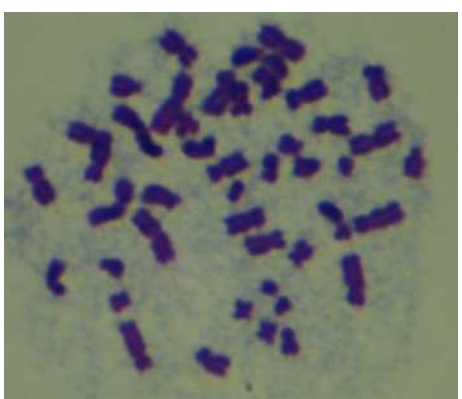

Fig 8. Case2 chromosomal study 


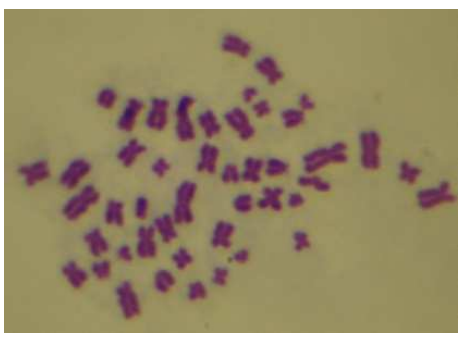

Fig 9. Case3 chromosomal study

\section{Discussion}

Congenital absence of the hand, Acheiria, is a rare defect occurring in 1:65 000 live births ${ }^{6}$. It is always unilateral and almost always sporadic; reports of Acheiria recurring in a family are sparse. Familial occurrence of Acheiria and forearm amputation has been recorded, in sibs, ${ }^{6}$ uncle and niece, uncle and nephew, and in a great grandmother and her great granddaughter. ${ }^{7}$ It have been reported also to be caused by the use of Hydantoin anticonvulsant for treatment of maternal epilepsy during pregnancy ${ }^{8}$.

In this report one of the patients ( case 3) have absence of his left hand $\&$ radius ( left Acheiria $\&$ radial hemimelia) ${ }^{8}$. Radial hemimelia is a congenital longitudinal deficiency of the radius bone of the forearm characterized by partial or total absence of the radius. It occurs in $1 / 30,000-100,000$ live births, and is slightly more common in males than in females (sex ratio of $3: 2$ ), Radial hemimelia is bilateral in $50 \%$ of cases and the right side is more commonly involved than the left (with a ratio of 2:1). Approximately $5-10 \%$ of radial hemimelia cases are familial but the etiology remains unknown $^{9}$. The deformities are believed to develop early in pregnancy, between the 28th and 56th day of gestation.

Maternal drug use (cocaine, thalidomide or valproate), compression of the uterus and vascular injury have been suggested to play a role in the etiopathogenesis of this condition, but these theories have not been proven. Diagnosis is based on clinical examination and $\mathrm{X}$ rays.

Radial hemimelia can be detected by prenatal ultrasound scan at the $20^{\text {th }}$ week of gestation ${ }^{9}$. Considering that the number of live births in our hospital during the year 2011 was 7230 , the incidence of Acheiria in our hospital is 4: 10.000 live births compared to the overall incidence of 1 : 65.000 livebirths \& the incidence of radial hemimelia is $0.5: 1000$ live births compared to the overall incidence of 1:30.000-100.000 live births.

The unilateralism of hemimelia in case 3 have been reported by many authors to occur as much as $80 \%$ of hemimelia \& confers better prognosis in terms of functionality ${ }^{10}$. Radial hemimelia is frequently associated with other congenital anomalies or syndromes, including chromosomal anomalies (trisomy 13, 18 and 21), HoltOram syndrome, Roberts syndrome, Rothmund-Thomson syndrome, thrombocytopenia-absent radius (TAR) syndrome (in which characteristically the thumb is present), Townes-Brocks syndrome, VACTERL association and Fanconi anemia ${ }^{10}$.
There were no anomalies detected in our 3 patients elsewhere in the body apart from Acheiria, and Acheiria \& radial hemimelia (case 3) by clinical examination, laboratory and radiological workup.

\section{Conclusions \& Recommendations}

The absence of contributory family and drug history, normal chromosomal study in the 3 cases, in addition to the non-specific linkage to any environmental factor lead us to the belief that this combination of abnormalities is of sporadic occurrence. These defects could be due to environmental contaminants which are known components of modern weaponry. Investigations of metal contaminants, and elucidation of the types and body burden of metals, combined with simultaneous registry of the population's reproductive history, will allow the identification of families at high risk and will facilitate therapeutic measures to remediate the damages.

\section{Consent}

Consent for publication was obtained from all the parents.

\section{Ethical Approval}

Ethical approval was granted by the scientific committee in Fallujah hospital. Authors have declared that no competing interests exist.

\section{References}

[1] J. Hib, Embriologya Médica, México: Editorial Interamericana - McGraw Hill, 1994

[2] Busby C, Hamdan M, Ariabi E. Cancer, infant mortality and birth sex-ratio in Fallujah, Iraq 2005-2009. Int J Environ Res Public Health. 2010;7:2828-37. http://doi.org/ck8j8z

[3] Alaani S, Savabieasfahani M, Tafash $M$ et al. Four polygamous families with congenital birth defects from Fallujah Iraq. Int J Environ Res Public Health. 2011;8:89-96. http://dx.doi.org/10.3390/ijerph8010089

[4] Alaani S, Tafash M, Busby C, et al. Uranium and other contaminants in hair from the parents of children with congenital anomalies in Fallujah, Iraq. Confl Health. 2011;5:15. http://dx.doi.org/10.1186/1752-1505-5-15

[5] Samira Alaani, MB.Ch.B, D.Ch1, Mohannad A.R. AlFallouji, PhD, FRCS (Edin), FRCS (Glas), FRCSI, PD (Law)1 Christopher Busby, BSc, PhD ; Malak Hamdan, M.Eng , Pilot Study of Congenital Anomaly Rates at Birth in Fallujah, Iraq, 2010, JIMA: Vol 44, 2012 , DOI: http://dx.doi.org/10.5915/44-1-10463

[6] Kohler HG. Congenital transverse defects of limbs and digits. Arch Dis Child 2001;37:263-76.

[7] Atlas of genetic diagnosis \& counseling, Harold Chen, MD, FAAP,FACMG,page 407. www.orphanet.com 

Year 2011 in Fallujah General Hospital

[8] Jain Sk, Lakhatkia PK. Profile of congenital transverse deficiencies among cases of

[9] Congenital orthopedic abnormalities. Journal of orthopedic surgery 2002,$10 ; 45-52$.
[10] Shafi M, Hui JHP. Common paediatric orthopedic problems in the upper limbs.

[11] Singapore Med J 2006;47:654-659. 